\title{
Acute necrotizing encephalopathy of childhood (ANEC)- Trilaminar sign on MRI
}

\author{
Mounisha Kethineni ${ }^{1}$, Venkatesh Manchikanti ${ }^{2 *}$ and Sundeep NVK ${ }^{3}$ \\ ${ }^{1}$ Postgraduate, Department of radiology, JJMMC, Davanagere, Karnataka, India \\ ${ }^{2}$ Assistant professor, Department of Radiology, Narayana medical college, Nellore, AP, India \\ ${ }^{3}$ Department of Radiodiagnosis, Government General Hospital and Medical college, Ongole, AP, India
}

\begin{abstract}
Acute necrotizing encephalopathy of childhood (ANEC) is a disease characterized by respiratory or gastrointestinal infection and high fever accompanying with rapid alteration of consciousness and seizures. We report a classic case with characteristic imaging findings on MRI which is crucial in diagnosing and of prognostic importance.
\end{abstract}

\section{Introduction}

Acute necrotising encephalopathy of childhood (ANEC) affects infants and children and can present with respiratory or gastrointestinal infection and high fever accompanying with rapid alteration of consciousness and seizures. ANEC is characterized by multiple, symmetrical lesions in the thalami, putamina, cerebral and cerebellar white matter, and brainstem tegmentum [1].

\section{Case report}

We present a case of a previously healthy 2 year old child which was referred to us with fever and altered sensorium since 1 day. Child was unable to take feeds and not responding to oral commands. MRI was done by using Philips achieva $1.5 \mathrm{~T}$ machine revealed multiple symmetrical T2 hyper intense areas(Figure 1) noted involving bilateral thalami capsular region, mid brain and pons showing areas of diffusion restriction with reduced ADC values and few foci of blooming on GRE sequence(Figure 2) (hemorrhagic foci). The classical neuroimaging of ANEC was "concentric or laminar structure" or "tricolour pattern" / target-like appearance (Figure 2), this typical manifestation is more obvious on ADC of MRI. On post contrast T1 weighted image (Figure 3) showed peripheral rim of contrast enhancement. All imaging features were suggestive of Acute necrotising encephalopathy. It is noteworthy that typical lesions appear predominantly in the grey matter, especially in the bilateral thalami. Child was admitted in PICU for 5 days and child was deteriorating, underwent intubation and was stabilized with inotropic support. Later child expired on $6^{\text {th }}$ day.

\section{Discussion}

The etiology and pathogenesis of Acute necrotising encephalopathy of childhood (ANEC) remains unknown. Although influenza A virus, mycoplasma, herpes simplex virus, and human herpes virus- 6 have been reported as common causative agents, it is now believed that this disease is most likely immune-mediated or metabolic. It has been reported that cytokines, such as tumor necrosis factor receptor-1, interleukin-1, and interleukin-6, could mediate the disease. The clinical course of ANEC

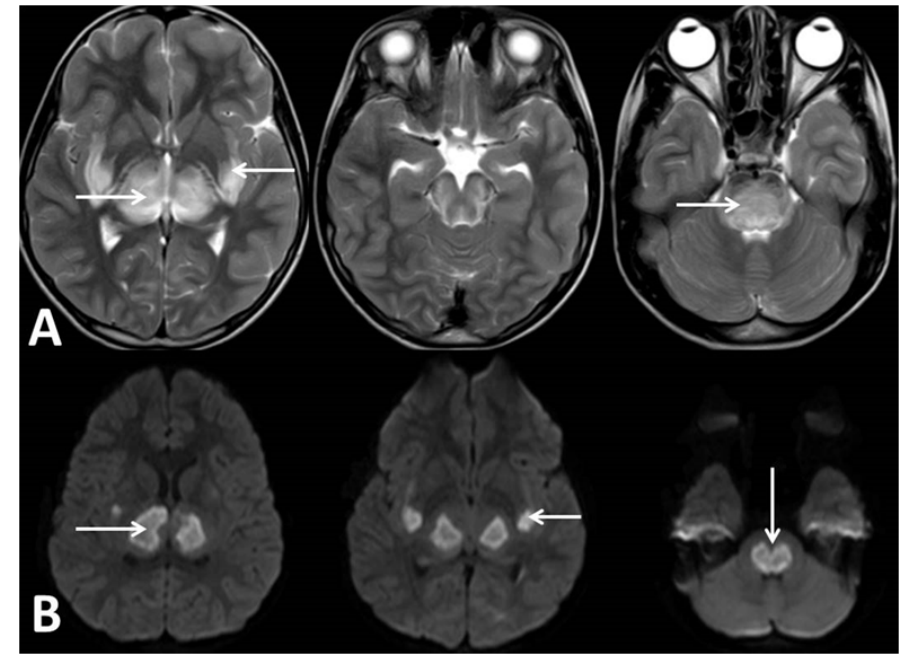

Figure 1. MRI T2 axial sections (A) showing multiple symmetrical hyperintense areas noted involving bilateral thalami capsular region, mid brain and pons with DWI Images (B) showing bilateral symmetrical areas of diffusion restriction

is fulminant, with a rapid onset of convulsions, impaired consciousness, vomiting, and variable degrees of hepatic dysfunction. Affected patients have high mortality and severe neurologic sequelae. From a pathologic perspective, the lesions show edema, petechial hemorrhage, and necrosis. Absence of inflammatory cells in affected brain parenchyma is characteristic, which differentiates this disease from the more common entities of acute disseminated encephalomyelitis and acute hemorrhagic encephalitis [1].

${ }^{*}$ Correspondence to: Venkatesh Manchikanti, Assistant professor, Department of Radiology, Narayana medical college, Nellore, AP, India, E-mail: drvenki143@gmail.com

Key words: acute necrotising encephalopathy, MRI, altered sensorium, fever

Received: April 30, 2020; Accepted: May 15, 2020; Published: May 20, 2020 


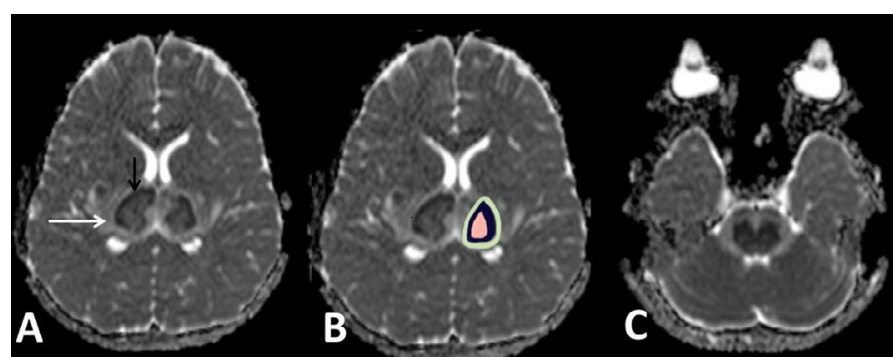

Figure 2. MRI ADC axial section images showing multiple symmetrical center hyper intense areas (hemorrhagic necrosis-Pink) with hypo intense rim (cytotoxic edema- purple) showing diffusion restriction which is again surrounded by hyper intense rim (vasogenic edema-Green) noted involving bilateral thalami capsular region, mid brain and pons

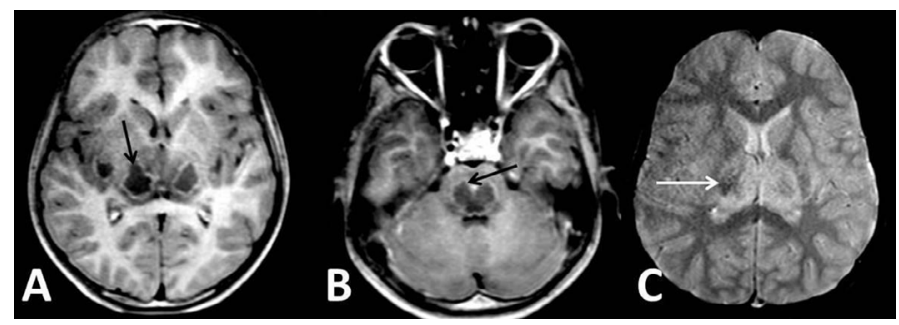

Figure 3. MRI GRE(C) and T1 post contrast(A and B) axial section images showing peripheral post contrast enhancement in bilateral thalamus and lentiform nucleus, midbrain, and pons

ANE is a progressive and devastating disease, regardless of treatment. The prognosis of ANE varies from complete recovery to death. It was estimated that the mortality rate was about $30 \%$ and less than $10 \%$ of patients recovered completely while the neurological sequelae were frequent in survivors [2-4].

The hallmark of neuroradiologic manifestations of ANE is multifocal, symmetric brain lesions involving both gray matter and white matter which are demonstrated by computed tomography (CT) or magnetic resonance imaging (MRI), i.e consistent with histopathologic findings via autopsy. The topographic distributions are remarkably similar in patients with ANE including thalami, brain stem, cerebral white matter, and cerebellum. Bilateral thalami are typically involved in all patients with ANE, which is a distinctive feature of ANE. Spinal cord may occasionally be involved as well. Lesions in the brain are edematous and combined with mass effect at the onset of ANE. Hypodensities are frequently seen on CT and homogeneously prolonged $\mathrm{T} 1$ and $\mathrm{T} 2$ relaxation time of the brain lesions on MRI are found in most patients. Moreover, the feature of restricted water diffusibility on diffusionweighted imaging (DWI) and apparent diffusion coefficient (ADC) can be found in majority of ANE patients with typical tricolour pattern in few patients. On the corresponding T1-weighted imaging (T1WI), increased signal intensities in the center surrounded by the decreased signals are detected, while T2-weighted imaging (T2WI) may reveal decreased signal intensities that are surrounded by increased or homogeneous increased signal intensities. Small petechial hemorrhage may usually obscure. The susceptibility weighted imaging (SWI) is more sensitive in showing the petechial hemorrhage of ANE, which demonstrate low signal intensities [5].

Gadolinium-contrast MRI has been useful in identifying lesions at the very early stage of ANE when conventional CT, MRI, even DWI and ADC show no abnormalities. This finding suggests that alteration of the BBB permeability might be the first step in the development of brain lesions. The gadolinium contrast MRI may therefore be very helpful for early diagnosis so as to initiate treatment as early as possible [5].

\section{Conclusion}

In conclusion, the diagnosis of ANE is mainly based on the clinical and radiologic features by exclusion of other resembling diseases. Although ANEC is a rare disease, it should not be under-diagnosed. MRI imaging shows classic features of trilaminar sign as described which can be of poor prognostic importance. The prognosis of ANE is variable; however, it is still a potentially devastating disease leading to death and severe neurological sequelae.

\section{References}

1. Wong AM, Simon EM, Zimmerman RA (2006) Acute necrotizing encephalopathy of childhood: correlation of MR findings and clinical outcome. Am J Neuroradiol 27: 1919-1923. [Crossref]

2. Mastroyianni SD, Voudris KA, Ktsarou E (2003) Acute necrotizing encephalopathy associated with parainfluenza virus in a Caucasian child. J Child Neurol 18: 570-572. [Crossref]

3. Lee JH, Lee M (2012) Recurrent acute necrotizing encephalopathy in a Korean child: the first non-caucasian case. J Child Neurol 27: 1343-1347. [Crossref]

4. Ravid S, Topper L, Eviatar L (2001) Acute necrotizing encephalopathy presenting as a basal ganglia syndrome. J Child Neurol 16: 461-462. [Crossref]

5. Xiujuan Wu, Wei Wu, Wei Pan (2015) Acute necrotizing encephalopathy: An underrecognized cinicoradiologic disorder. Mediators Inflamm 10: 1-10. [Crossref]

Copyright: $@ 2020$ Kethineni M. This is an open-access article distributed under the terms of the Creative Commons Attribution License, which permits unrestricted use, distribution, and reproduction in any medium, provided the original author and source are credited. 